Brazilian Journal
of Chemical
Engineering

ISSN 0104-6632

Printed in Brazil

www.abeq.org.br/bjche

Vol. 28, No. 02, pp. 325 - 332, April - June, 2011

\title{
VAPOR-LIQUID EQUILIBRIUM MEASUREMENTS FOR THE BINARY SYSTEM METHYL ACETATE+ETHANOL AT 0.3 AND $0.7 \mathrm{MPa}$
}

\author{
P. Susial*, R. Rios-Santana and A. Sosa-Rosario \\ Escuela Técnica Superior de Ingenieros Industriales, Phone: 928-458658, \\ Universidad de Las Palmas de Gran Canaria, 35017 Tafira Baja, \\ Las Palmas de Gran Canaria - Islas Canarias, España. \\ E-mail: psusial@dip.ulpgc.es
}

(Submitted: February 3, 2010 ; Revised: February 7, 2011 ; Accepted: February 11, 2011)

\begin{abstract}
This paper reports experimental data of the isobaric vapor-liquid equilibria (VLE) for the mixture methyl acetate + ethanol at 0.3 and $0.7 \mathrm{MPa}$, determined using a copper still ebulliometer. The activity coefficients obtained from the experimental data were correlated by using different thermodynamic mathematical models. All the binary systems show a positive consistency when subjected to the point-to-point test of Van Ness. The prediction of VLE data obtained with the UNIFAC and ASOG methods has been verified with experimental data.

Keywords: VLE isobaric data; Activity coefficient; Methyl ester; Ethanol.
\end{abstract}

\section{INTRODUCTION}

Previous papers (Ortega and Susial, 1990; Ortega and Susial, 1993) have reported VLE data for methyl esters and alkanols at different pressures. The methyl acetate + ethanol binary system was reported at isothermal conditions of $323.15 \mathrm{~K}, 333.15 \mathrm{~K}, 343.15 \mathrm{~K}$ and $353.15 \mathrm{~K}$ by Perelygin and Volkov (1970) (see Gmehling and Onken, 1986). This system has also been reported at isobaric conditions by Perelygin and Volkov (1970) and Nishi (1972) (see Gmehling and Onken, 1986) and by Ortega et al. (1990) at $101.3 \mathrm{kPa}$, while Ortega and Susial (1990) have published data for the methyl acetate + ethanol system at $114.66 \mathrm{kPa}$ and $127.99 \mathrm{kPa}$. In the present work, VLE measurements of methyl acetate + ethanol at $0.1,0.3$ and $0.7 \mathrm{MPa}$ obtained with a copper still are presented.

The experimental data of this paper at $0.1,0.3$ and $0.7 \mathrm{MPa}$ were verified thermodynamically using the point-to-point test of Van Ness (Van Ness et al., 1973) and by applying the procedure of Barker
(Barker, 1953) implemented in the computer program of Fredenslund et al. (1977).

All data included in this work have passed the consistency test according to the Fredenslund criteria (Fredenslund et al., 1977). These data were used to check the reliability of UNIFAC (Fredenslund et al., 1977; Fredenslund et al., 1975; Gmehling et al., 1993; Larsen et al., 1987) and ASOG (Kojima and Tochigi, 1979) group contribution models in order to expand the applicability of these predictive models.

\section{EXPERIMENTAL}

\section{Materials and Apparatus}

In this paper, ethanol and methyl acetate, both from Panreac Química S.A., were used as received. The purity and the experimental values of density, refractive index and normal boiling point of these products are shown in Table 1 . The normal boiling

*To whom correspondence should be addressed 
point at $0.1 \mathrm{MPa}$ was determined with the copper ebulliometer. A Kyoto Electronics DA-300 vibrating tube density meter with an uncertainty of $\pm 0.1 \mathrm{~kg} \cdot \mathrm{m}^{-3}$ and a Zusi model 315RS Abbe refractometer with an uncertainty of \pm 0.0002 units were used for density and refractive index determinations, respectively. The measures of the pure substance properties are compared with literature values.

\section{VLE Equipment and Procedure}

A still ebulliometer developed and built in copper, with all its different pieces assembled with silver-weldings is presented in Figure 1. The still has a configuration similar to the apparatus made of glass by de Afonso et al. (1983). The equipment has a double-walled inverted vessel where a boiling mixture of liquid and vapor is generated by an electric heater. This boiling mixture rises in a tube that works like a Cotrell pump. The Cotrell tube is connected to a vessel that acts like an equilibrium chamber. The liquid and vapor phases are carried out on separate paths from this equilibrium chamber. A tube connected to a funnel collects and circulates the liquid phase in the equipment. This tube has a lateral path that ends in a valve. The vapor phase is carried out to the cooler. The cooler has a coaxial tube that returns the condensate to the still and it has a lateral path with a finishing valve. Therefore, both phases are refluxed.

The equilibrium equipment has been designed to work at moderate pressures. Therefore, slight modifications were made to this copper still in order to ease sampling of both phases at the working pressures (see Figure 1). Some airtight outer vessels must be used. In addition, it was necessary to prepare J-type thermocouples with a ring silver-soldered to both its cover and the ebulliometer. Thermocouples of Thermocoax (range $1073 \mathrm{~K}$ ) with $0.1 \%$ precision were used for the temperature measurements. The working pressure was measured with a NuovaFima manometer with a range from 0 to $1.0 \mathrm{MPa}$ and $1 \%$ precision.

Table 1: Physical properties of pure compounds at atmospheric pressure

\begin{tabular}{|c|c|c|c|c|c|c|c|}
\hline & \multirow{2}{*}{ purity/mass $\%$} & \multicolumn{2}{|c|}{$\mathbf{T}_{\mathbf{b p}} / \mathrm{K}$} & \multicolumn{2}{|c|}{$\rho(298.15 \mathrm{~K}) /\left(\mathrm{kg}^{\prime} \mathrm{m}^{-3}\right)$} & \multicolumn{2}{|c|}{ nD (298.15 K) } \\
\hline & & $\exp$ & lit & $\exp$ & lit & $\exp$ & lit \\
\hline ethanol & puriss. p.a. $>99.9$ & 351.35 & $\begin{array}{r}351.443(\mathrm{~A}) \\
351.45(\mathrm{~B}) \\
351.44(\mathrm{C})\end{array}$ & 785.2 & $\begin{array}{r}784.93(\mathrm{~A}) \\
785.2(\mathrm{~B})\end{array}$ & 1.3600 & $\begin{array}{r}1.35941(\mathrm{~A}) \\
1.3594(\mathrm{~B}, \mathrm{C})\end{array}$ \\
\hline methyl acetate & puriss. $>99$ & 330.05 & $\begin{array}{r}330.018(\mathrm{~A}) \\
329.95(\mathrm{~B}) \\
330.09(\mathrm{C})\end{array}$ & 927.3 & $\begin{array}{l}927.90(\mathrm{~A}) \\
927.30(\mathrm{~B})\end{array}$ & 1.3590 & $\begin{array}{r}1.3589(\mathrm{~A}, \mathrm{C}) \\
1.3587(\mathrm{~B})\end{array}$ \\
\hline
\end{tabular}

(A) Riddick et al., 1986; (B) Nagata et al., 1976; (C) Yaws, 2003

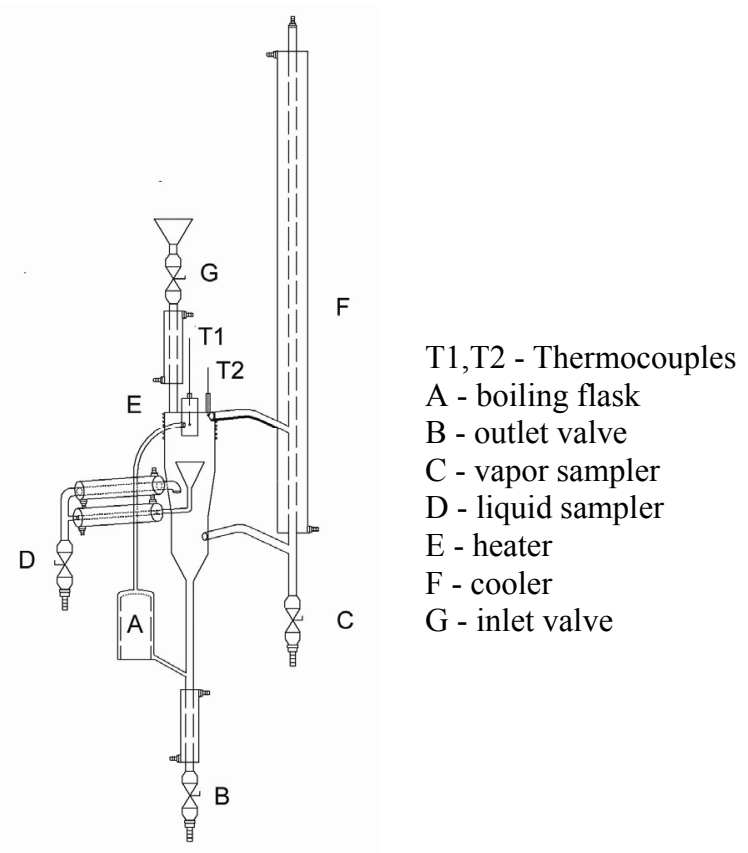

Figure 1: Equilibrium recirculation still used for the VLE measurements. 
The copper still described in this paper showed good agreement with the ebulliometer previously used, the operation procedure also being similar (de Afonso et al., 1983; Ortega et al., 1990; Susial et al., 1989). The procedure differs in the residence time of the mixture inside the ebulliometer and the sampling method. The ebulliometer has a $400 \mathrm{~cm}^{3}$ mixture capacity, the reason why a 90 min recirculation time is necessary for the mixture. This was evaluated by a previous test of operation time vs. mole fraction of both phases (de Afonso et al., 1983). Samples of the liquid and vapor phases were removed from the airtight vessels with syringes and the compositions of the equilibrium phases were obtained by densimetry at $298.15 \mathrm{~K}$, using a composition-density standard curve prepared for the mixture studied in this paper,

$\rho=785.2+181.1 \cdot x_{1}-51.5 \cdot x_{1}^{2}+12.5 \cdot x_{1}^{3}$

As in previous works (Ortega et al., 1990; Ortega and Susial, 1990; Susial et al., 1989), the values of the data pairs composition-density were correlated by using the Nelder and Mead (1967) procedure. Therefore, considering the different apparatus used, the estimated accuracy in the determination of both the liquid and vapor phase was better than \pm 0.002 units of mole fraction.

\section{RESULTS AND TREATMENT}

With the copper still ebulliometer, the experimental $\mathrm{T}-\mathrm{x}_{1}-\mathrm{y}_{1}$ data (Table 2) for methyl acetate+ethanol were obtained at $0.1,0.3$ and $0.7 \mathrm{MPa}$. Also included in Table 2 are the activity coefficients of the liquid phase for each system, calculated by using the following equation:

$\gamma_{i}=\frac{\varphi_{i} y_{i} p}{x_{i} \varphi_{i}^{o} p_{i}^{o}} \exp \left[\frac{\left(p_{i}^{o}-p\right) v_{i}^{L}}{R T}\right]$

The fugacity coefficients were calculated by using the virial state equation truncated at the second term, and from the following equation:

$\varphi_{i}=\exp \left[\frac{p}{R T}\left(2 \sum_{j} y_{i} B_{i j}-\sum_{i} \sum_{j} y_{i} y_{j} B_{i j}\right)\right]$

The second virial coefficients were calculated by the Hayden and O'Connell (1975) method. The liquid molar volumes of the pure compounds were estimated by the equation of Yen and Woods (1966).

VLE experimental data in this paper were verified by using the thermodynamic consistency test of Van Ness et al. (1973). The proposed computer program of Fredenslund et al., (1977) was applied using physical properties from the literature (Fredenslund et al., 1977; Riddick et al., 1986; Yaws, 2003) and the correlations of the vapor pressures from a previous paper (Susial et al., 2010).

All the systems included in this work passed the point-to-point test because they have an average deviation of $\delta\left(\mathrm{y}_{1}\right)<0.01$ (Fredenslund et al., 1977). The experimental data (Table 2 ) were correlated by using the calculated activity coefficients in the form of $\mathrm{G}^{\mathrm{E}} / \mathrm{RT}$ vs. $\mathrm{x}_{1}$ with the Redlich-Kister, Van Laar, Margüles, Wilson, NRTL and UNIQUAC models. To calculate the constants of each model, a non-linear regression procedure was employed (Nelder and Mead, 1967), considering a minimization of the objective function (OF) (Holmes and Winkle, 1970; Postigo et al., 2009):

$\mathrm{OF}=\sum_{1}^{\mathrm{n}}\left(\gamma_{1}^{\exp }-\gamma_{1}^{\text {calc }}\right)^{2}+\sum_{1}^{\mathrm{n}}\left(\gamma_{2}^{\exp }-\gamma_{2}^{\text {calc }}\right)^{2}$

The results of the correlations with thermodynamic mathematical models are presented in Table 3. The models give good fit to the experimental data and show acceptable mean deviations for the prediction of the vapor phase composition.

In addition, as in previous papers (Ortega et al., 1990; Ortega and Susial, 1990; Susial et al., 1989), the experimental data of each system were correlated to a fitting function (FF) with a polynomial form (see Table 4). Results of the experimental data treatment with the different FF, using the Nelder and Mead (1967) procedure to minimize the summation of the square of the deviations, are shown in Table 4.

A mathematical treatment similar to literature data (Ortega et al., 1990; Ortega and Susial, 1990) was made for the parameter of interest $\left(\mathrm{y}_{1}-\mathrm{x}_{1}\right)$ or $\mathrm{T}$. The fits of the experimental data of this paper (Table 4) are plotted in Figure 2 and 3, respectively).

The examination of the equilibrium data obtained in this work shows a good variation with pressure (see Figure 2) and an appreciable relation can be also observed between our systems at $0.1,0.3$ and 0.7 $\mathrm{MPa}$ and literature data at $127.99 \mathrm{kPa}$ (Ortega and Susial, 1990). In Figure 2, some differences are seen between data from this study at $0.1 \mathrm{MPa}$ and the data from literature at $101.32 \mathrm{kPa}$ (Ortega et al., 1990), but these differences are also present between the data obtained by Ortega et al. (1990) at $101.32 \mathrm{kPa}$ and literature data at $127.99 \mathrm{kPa}$ (Ortega and Susial, 1990). The above mentioned relationship between the data from this work and literature data at $127.99 \mathrm{kPa}$ (Ortega and Susial, 1990) is shown in the inset in Figure 3. On the other hand, the differences observed in Figure 3 between data from this paper at $0.1 \mathrm{MPa}$ and literature data at $101.32 \mathrm{kPa}$ (Ortega et al., 1990) may be due to the greater uncertainty in the determination of temperature in this work. 
Table 2: VLE experimental data and calculated values of activity coefficients of the liquid phase

\begin{tabular}{|c|c|c|c|c|}
\hline $\mathbf{T} / \mathbf{K}$ & $\mathbf{x}_{1}$ & $\mathbf{y}_{1}$ & $\gamma_{1}$ & $\gamma_{2}$ \\
\hline \multicolumn{5}{|c|}{ methyl acetate (1) + ethanol (2) at $0.1 \mathrm{MPa}$} \\
\hline 351.35 & 0.000 & 0.000 & & 1.000 \\
\hline 349.05 & 0.038 & 0.127 & 1.789 & 0.979 \\
\hline 345.95 & 0.079 & 0.254 & 1.892 & 0.991 \\
\hline 341.35 & 0.168 & 0.418 & 1.691 & 1.037 \\
\hline 339.75 & 0.211 & 0.496 & 1.681 & 1.014 \\
\hline 337.25 & 0.291 & 0.577 & 1.538 & 1.056 \\
\hline 336.75 & 0.335 & 0.602 & 1.417 & 1.083 \\
\hline 336.05 & 0.372 & 0.635 & 1.377 & 1.084 \\
\hline 334.45 & 0.441 & 0.686 & 1.323 & 1.125 \\
\hline 333.75 & 0.488 & 0.703 & 1.254 & 1.199 \\
\hline 332.95 & 0.569 & 0.738 & 1.160 & 1.302 \\
\hline 331.95 & 0.643 & 0.773 & 1.112 & 1.425 \\
\hline 331.55 & 0.686 & 0.791 & 1.081 & 1.519 \\
\hline 331.15 & 0.720 & 0.809 & 1.068 & 1.586 \\
\hline 330.45 & 0.855 & 0.874 & 0.995 & 2.085 \\
\hline 330.25 & 0.874 & 0.892 & 1.000 & 2.075 \\
\hline 330.15 & 0.902 & 0.911 & 0.993 & 2.208 \\
\hline 330.15 & 0.911 & 0.921 & 0.994 & 2.158 \\
\hline 329.95 & 0.959 & 0.959 & 0.990 & 2.453 \\
\hline 330.05 & 1.000 & 1.000 & 1.000 & \\
\hline \multicolumn{5}{|c|}{ methyl acetate (1) + ethanol $(2)$ at $0.3 \mathrm{MPa}$} \\
\hline 382.05 & 0.000 & 0.000 & & 1.000 \\
\hline 380.65 & 0.018 & 0.064 & 2.387 & 0.985 \\
\hline 378.65 & 0.058 & 0.162 & 1.972 & 0.980 \\
\hline 375.15 & 0.141 & 0.313 & 1.715 & 0.989 \\
\hline 374.15 & 0.168 & 0.350 & 1.652 & 0.999 \\
\hline 372.35 & 0.225 & 0.418 & 1.544 & 1.020 \\
\hline 371.45 & 0.254 & 0.449 & 1.504 & 1.034 \\
\hline 370.45 & 0.291 & 0.481 & 1.444 & 1.061 \\
\hline 370.35 & 0.320 & 0.512 & 1.402 & 1.043 \\
\hline 367.75 & 0.465 & 0.610 & 1.233 & 1.159 \\
\hline 367.45 & 0.496 & 0.635 & 1.213 & 1.164 \\
\hline 366.95 & 0.536 & 0.652 & 1.168 & 1.226 \\
\hline 365.65 & 0.627 & 0.703 & 1.115 & 1.363 \\
\hline 365.05 & 0.703 & 0.756 & 1.088 & 1.436 \\
\hline 364.15 & 0.892 & 0.892 & 1.037 & 1.802 \\
\hline 364.45 & 0.950 & 0.940 & 1.018 & 2.138 \\
\hline 364.55 & 0.979 & 0.969 & 1.015 & 2.619 \\
\hline 365.25 & 1.000 & 1.000 & 1.000 & \\
\hline \multicolumn{5}{|c|}{ methyl acetate (1) + ethanol (2) at $0.7 \mathrm{MPa}$} \\
\hline 410.75 & 0.000 & 0.000 & & 1.000 \\
\hline 410.05 & 0.031 & 0.052 & 1.328 & 0.992 \\
\hline 409.05 & 0.045 & 0.092 & 1.653 & 0.990 \\
\hline 407.65 & 0.092 & 0.168 & 1.521 & 0.991 \\
\hline 407.15 & 0.113 & 0.204 & 1.520 & 0.983 \\
\hline 406.35 & 0.141 & 0.247 & 1.500 & 0.981 \\
\hline 404.55 & 0.197 & 0.312 & 1.410 & 1.007 \\
\hline 404.05 & 0.232 & 0.350 & 1.358 & 1.009 \\
\hline 402.95 & 0.298 & 0.410 & 1.269 & 1.032 \\
\hline 401.65 & 0.388 & 0.481 & 1.177 & 1.080 \\
\hline 399.95 & 0.504 & 0.577 & 1.129 & 1.138 \\
\hline 399.35 & 0.561 & 0.618 & 1.101 & 1.181 \\
\hline 398.25 & 0.686 & 0.703 & 1.050 & 1.324 \\
\hline 398.25 & 0.809 & 0.791 & 1.002 & 1.531 \\
\hline 398.65 & 0.911 & 0.892 & 0.994 & 1.677 \\
\hline 399.15 & 0.989 & 0.979 & 0.994 & 2.600 \\
\hline 399.35 & 1.000 & 1.000 & 1.000 & \\
\hline
\end{tabular}


Table 3: Correlation parameters of $G^{\mathrm{E}} / \mathrm{RT}$ vs. $\mathrm{x}_{1}$, mean deviations and standard deviations

\begin{tabular}{|c|c|c|c|c|c|c|}
\hline Model & \multicolumn{2}{|c|}{ parameters } & $\delta\left(\mathbf{y}_{1}\right)$ & $\sigma\left(\gamma_{1}\right)$ & $\sigma\left(\gamma_{2}\right)$ & $\sigma\left(G^{\mathrm{E}} / \mathbf{R T}\right)$ \\
\hline \multicolumn{7}{|c|}{ methyl acetate (1) + ethanol (2) at $0.1 \mathrm{MPa}$} \\
\hline Redlich-Kister & $\mathrm{A}_{0}=0.843$ & $\mathrm{~A}_{1}=0.160$ & 0.007 & 0.05 & 0.05 & 0.01 \\
\hline Van Laar & $\mathrm{A}_{12}=0.712$ & $\mathrm{~A}_{21}=1.019$ & 0.007 & 0.06 & 0.05 & 0.01 \\
\hline Margules & $A_{12}=0.671$ & $\mathrm{~A}_{21}=0.896$ & 0.008 & 0.04 & 0.12 & 0.01 \\
\hline Wilson & $\Delta \lambda_{12}=1253.4\left(\mathrm{~J} \bullet \mathrm{mol}^{-1}\right)$ & $\Delta \lambda_{21}=1605.0\left(\mathrm{~J} \cdot \mathrm{mol}^{-1}\right)$ & 0.006 & 0.05 & 0.05 & 0.01 \\
\hline $\operatorname{NRTL}(\alpha=0.47)$ & $\Delta \mathrm{g}_{12}=2489.7\left(\mathrm{~J} \bullet \mathrm{mol}^{-1}\right)$ & $\Delta \mathrm{g}_{21}=366.6\left(\mathrm{~J} \cdot \mathrm{mol}^{-1}\right)$ & 0.007 & 0.05 & 0.05 & 0.01 \\
\hline UNIQUAC $(Z=10)$ & $\Delta \mathrm{u}_{12}=2316.8\left(\mathrm{~J} \bullet \mathrm{mol}^{-1}\right)$ & $\Delta \mathrm{u}_{21}=-744.0\left(\mathrm{~J} \bullet \mathrm{mol}^{-1}\right)$ & 0.007 & 0.05 & 0.05 & 0.01 \\
\hline \multicolumn{7}{|c|}{ methyl acetate (1) + ethanol (2) at $0.3 \mathrm{MPa}$} \\
\hline Redlich-Kister & $\mathrm{A}_{0}=0.806$ & $\mathrm{~A}_{1}=0.048$ & 0.005 & 0.09 & 0.011 & 0.02 \\
\hline Van Laar & $\mathrm{A}_{12}=0.751$ & $\mathrm{~A}_{21}=0.865$ & 0.005 & 0.10 & 0.10 & 0.02 \\
\hline Margules & $\mathrm{A}_{12}=0.820$ & $\mathrm{~A}_{21}=0.667$ & 0.006 & 0.06 & 0.21 & 0.02 \\
\hline Wilson & $\Delta \lambda_{12}=1927.9\left(\mathrm{~J} \bullet \mathrm{mol}^{-1}\right)$ & $\Delta \lambda_{21}=880.9\left(\mathrm{~J} \bullet \mathrm{mol}^{-1}\right)$ & 0.005 & 0.09 & 0.10 & 0.02 \\
\hline $\operatorname{NRTL}(\alpha=0.47)$ & $\Delta \mathrm{g}_{12}=1800.2\left(\mathrm{~J} \cdot \mathrm{mol}^{-1}\right)$ & $\Delta \mathrm{g}_{21}=1084.1\left(\mathrm{~J} \cdot \mathrm{mol}^{-1}\right)$ & 0.006 & 0.09 & 0.10 & 0.03 \\
\hline UNIQUAC $(Z=10)$ & $\Delta \mathrm{u}_{12}=1836.6\left(\mathrm{~J} \bullet \mathrm{mol}^{-1}\right)$ & $\Delta \mathrm{u}_{21}=-419.8\left(\mathrm{~J} \cdot \mathrm{mol}^{-1}\right)$ & 0.005 & 0.10 & 0.10 & 0.02 \\
\hline \multicolumn{7}{|c|}{ methyl acetate (1) + ethanol (2) at $0.7 \mathrm{MPa}$} \\
\hline Redlich-Kister & $\mathrm{A}_{0}=0.607$ & $\mathrm{~A}_{1}=0.205$ & 0.012 & 0.09 & 0.12 & 0.02 \\
\hline Van Laar & $\mathrm{A}_{12}=0.426$ & $\mathrm{~A}_{21}=0.899$ & 0.012 & 0.09 & 0.09 & 0.02 \\
\hline Margules & $\mathrm{A}_{12}=0.441$ & $\mathrm{~A}_{21}=0.570$ & 0.009 & 0.08 & 0.24 & 0.01 \\
\hline Wilson & $\Delta \lambda_{12}=63.9\left(\mathrm{~J} \bullet \mathrm{mol}^{-1}\right)$ & $\Delta \lambda_{21}=3076.4\left(\mathrm{~J} \cdot \mathrm{mol}^{-1}\right)$ & 0.012 & 0.09 & 0.09 & 0.02 \\
\hline $\operatorname{NRTL}(\alpha=0.47)$ & $\Delta \mathrm{g}_{12}=4244.0\left(\mathrm{~J} \bullet \mathrm{mol}^{-1}\right)$ & $\Delta \mathrm{g}_{21}=-970.3\left(\mathrm{~J} \cdot \mathrm{mol}^{-1}\right)$ & 0.014 & 0.10 & 0.08 & 0.02 \\
\hline UNIQUAC $(Z=10)$ & $\Delta \mathrm{u}_{12}=3604.5\left(\mathrm{~J} \cdot \mathrm{mol}^{-1}\right)$ & $\Delta \mathrm{u}_{21}=-1607.4\left(\mathrm{~J} \cdot \mathrm{mol}^{-1}\right)$ & 0.012 & 0.09 & 0.10 & 0.02 \\
\hline
\end{tabular}

Table 4: Fittings coefficients for FF and standard deviations

\begin{tabular}{|c|c|c|c|c|c|c|}
\hline $\mathbf{F F}$ & $\mathbf{R}_{\mathbf{T}}$ & $\overline{\mathbf{A}_{0}}$ & $\mathbf{A}_{1}$ & $\mathbf{A}_{2}$ & $\mathbf{\mathbf { A } _ { 3 }}$ & \\
\hline \multicolumn{7}{|c|}{ methyl acetate (1) + ethanol (2) at $0.1 \mathrm{MPa}$} \\
\hline$\left(\mathrm{y}_{1}-\mathrm{x}_{1}\right)\left[\mathrm{x}_{1}\left(1-\mathrm{x}_{1}\right)\right]^{-1}=\sum_{\mathrm{k}=0} \mathrm{~A}_{\mathrm{k}}\left\{\mathrm{x}_{1}\left[\mathrm{x}_{1}+\mathrm{R}_{\mathrm{T}}\left(1-\mathrm{x}_{1}\right)\right]^{-1}\right\}^{\mathrm{k}}$ & 0.758 & 2.865 & -5.896 & 5.620 & -2.677 & $\sigma\left(\mathrm{y}_{1}-\mathrm{x}_{1}\right)<0.01$ \\
\hline$\left[\mathrm{T}-\mathrm{x}_{1} \mathrm{~T}_{\mathrm{bp} 1}-\left(1-\mathrm{x}_{1}\right) \mathrm{T}_{\mathrm{bp} 2}\right]\left[\mathrm{x}_{1}\left(1-\mathrm{x}_{1}\right)\right]^{-1}=\sum_{\mathrm{k}=0} \mathrm{~A}_{\mathrm{k}}\left\{\mathrm{x}_{1}\left[\mathrm{x}_{1}+\mathrm{R}_{\mathrm{T}}\left(1-\mathrm{x}_{1}\right)\right]^{-1}\right\}^{\mathrm{k}}$ & 0.267 & -62.708 & 43.235 & & & $\sigma(\mathrm{T}) / \mathrm{K}=0.25$ \\
\hline$\left[\mathrm{T}-\mathrm{y}_{1} \mathrm{~T}_{\mathrm{bp} 1}-\left(1-\mathrm{y}_{1}\right) \mathrm{T}_{\mathrm{bp} 2}\right]\left[\mathrm{y}_{1}\left(1-\mathrm{y}_{1}\right)\right]^{-1}=\sum_{\mathrm{k}=0} \mathrm{~A}_{\mathrm{k}}\left\{\mathrm{x}_{1}\left[\mathrm{x}_{1}+\mathrm{R}_{\mathrm{T}}\left(1-\mathrm{x}_{1}\right)\right]^{-1}\right\}^{\mathrm{k}}$ & 2.807 & 4.393 & -35.974 & & & $\sigma(\mathrm{T}) / \mathrm{K}=0.22$ \\
\hline$\left(G^{\mathrm{E}} / \mathrm{RT}\right)\left[\mathrm{x}_{1}\left(1-\mathrm{x}_{1}\right)\right]^{-1}=\sum_{\mathrm{k}=0} \mathrm{~A}_{\mathrm{k}}\left\{\mathrm{x}_{1}\left[\mathrm{x}_{1}+\mathrm{R}_{\mathrm{T}}\left(1-\mathrm{x}_{1}\right)\right]^{-1}\right\}^{\mathrm{k}}$ & -0.018 & 1.764 & -0.939 & & & $\sigma\left(\mathrm{G}^{\mathrm{E}} / \mathrm{RT}\right)<0.01$ \\
\hline \multicolumn{7}{|c|}{ methyl acetate (1) + ethanol (2) at $0.3 \mathrm{MPa}$} \\
\hline$\left(\mathrm{y}_{1}-\mathrm{x}_{1}\right)\left[\mathrm{x}_{1}\left(1-\mathrm{x}_{1}\right)\right]^{-1}=\sum_{\mathrm{k}=0} \mathrm{~A}_{\mathrm{k}}\left\{\mathrm{x}_{1}\left[\mathrm{x}_{1}+\mathrm{R}_{\mathrm{T}}\left(1-\mathrm{x}_{1}\right)\right]^{-1}\right\}^{\mathrm{k}}$ & 0.344 & 2.621 & -5.178 & 5.505 & -3.107 & $\sigma\left(\mathrm{y}_{1}-\mathrm{x}_{1}\right)<0.01$ \\
\hline$\left[\mathrm{T}-\mathrm{x}_{1} \mathrm{~T}_{\mathrm{bp} 1}-\left(1-\mathrm{x}_{1}\right) \mathrm{T}_{\mathrm{bp} 2}\right]\left[\mathrm{x}_{1}\left(1-\mathrm{x}_{1}\right)\right]^{-1}=\sum_{\mathrm{k}=0} \mathrm{~A}_{\mathrm{k}}\left\{\mathrm{x}_{1}\left[\mathrm{x}_{1}+\mathrm{R}_{\mathrm{T}}\left(1-\mathrm{x}_{1}\right)\right]^{-1}\right\}^{\mathrm{k}}$ & 1.226 & -46.930 & 80.540 & -71.950 & & $\sigma(\mathrm{T}) / \mathrm{K}=0.17$ \\
\hline$\left[\mathrm{T}-\mathrm{y}_{1} \mathrm{~T}_{\mathrm{bp} 1}-\left(1-\mathrm{y}_{1}\right) \mathrm{T}_{\mathrm{bp} 2}\right]\left[\mathrm{y}_{1}\left(1-\mathrm{y}_{1}\right)\right]^{-1}=\sum_{\mathrm{k}=0} \mathrm{~A}_{\mathrm{k}}\left\{\mathrm{x}_{1}\left[\mathrm{x}_{1}+\mathrm{R}_{\mathrm{T}}\left(1-\mathrm{x}_{1}\right)\right]^{-1}\right\}^{\mathrm{k}}$ & 2.285 & -2.199 & -36.419 & & & $\sigma(\mathrm{T}) / \mathrm{K}=0.18$ \\
\hline$\left(G^{\mathrm{E}} / \mathrm{RT}\right)\left[\mathrm{x}_{1}\left(1-\mathrm{x}_{1}\right)\right]^{-1}=\sum_{\mathrm{k}=0} \mathrm{~A}_{\mathrm{k}}\left\{\mathrm{x}_{1}\left[\mathrm{x}_{1}+\mathrm{R}_{\mathrm{T}}\left(1-\mathrm{x}_{1}\right)\right]^{-1}\right\}^{\mathrm{k}}$ & 6.640 & 0.608 & 0.768 & & & $\sigma\left(\mathrm{G}^{\mathrm{E}} / \mathrm{RT}\right)=0.01$ \\
\hline \multicolumn{7}{|c|}{ methyl acetate (1) + ethanol (2) at $0.7 \mathrm{MPa}$} \\
\hline$\left(\mathrm{y}_{1}-\mathrm{x}_{1}\right)\left[\mathrm{x}_{1}\left(1-\mathrm{x}_{1}\right)\right]^{-1}=\sum_{\mathrm{k}=0} \mathrm{~A}_{\mathrm{k}}\left\{\mathrm{x}_{1}\left[\mathrm{x}_{1}+\mathrm{R}_{\mathrm{T}}\left(1-\mathrm{x}_{1}\right)\right]^{-1}\right\}^{\mathrm{k}}$ & 1.181 & 1.180 & -3.331 & 4.182 & -2.557 & $\sigma\left(\mathrm{y}_{1}-\mathrm{x}_{1}\right)<0.01$ \\
\hline$\left[\mathrm{T}-\mathrm{x}_{1} \mathrm{~T}_{\mathrm{bp} 1}-\left(1-\mathrm{x}_{1}\right) \mathrm{T}_{\mathrm{bp} 2}\right]\left[\mathrm{x}_{1}\left(1-\mathrm{x}_{1}\right)\right]^{-1}=\sum_{\mathrm{k}=0} \mathrm{~A}_{\mathrm{k}}\left\{\mathrm{x}_{1}\left[\mathrm{x}_{1}+\mathrm{R}_{\mathrm{T}}\left(1-\mathrm{x}_{1}\right)\right]^{-1}\right\}^{\mathrm{k}}$ & 0.239 & -9.833 & -98.281 & 199.718 & -116.830 & $\sigma(\mathrm{T}) / \mathrm{K}=0.18$ \\
\hline$\left[\mathrm{T}-\mathrm{y}_{1} \mathrm{~T}_{\mathrm{bp} 1}-\left(1-\mathrm{y}_{1}\right) \mathrm{T}_{\mathrm{bp} 2}\right]\left[\mathrm{y}_{1}\left(1-\mathrm{y}_{1}\right)\right]^{-1}=\sum_{\mathrm{k}=0} \mathrm{~A}_{\mathrm{k}}\left\{\mathrm{x}_{1}\left[\mathrm{x}_{1}+\mathrm{R}_{\mathrm{T}}\left(1-\mathrm{x}_{1}\right)\right]^{-1}\right\}^{\mathrm{k}}$ & 2.864 & -4.576 & -53.990 & 39.147 & & $\sigma(\mathrm{T}) / \mathrm{K}=0.18$ \\
\hline$\left(G^{E} / R T\right)\left[x_{1}\left(1-x_{1}\right)\right]^{-1}=\sum_{k=0} A_{k}\left\{x_{1}\left[x_{1}+R_{T}\left(1-x_{1}\right)\right]^{-1}\right\}^{k}$ & 0.121 & 0.124 & 0.429 & & & $\sigma\left(\mathrm{G}^{\mathrm{E}} / \mathrm{RT}\right)=0.01$ \\
\hline
\end{tabular}




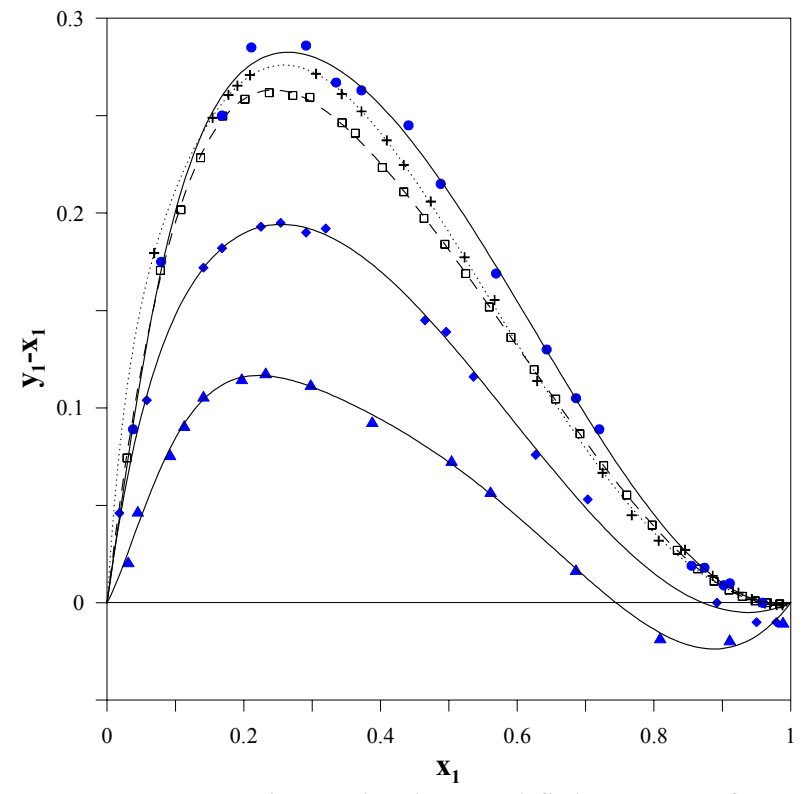

Figure 2: Experimental values and fitting curves for the mixture of methyl acetate (1) + ethanol (2) at 0.1 $\mathrm{MPa}(\bullet), 0.3 \mathrm{MPa}(\bullet)$ and $0.7 \mathrm{MPa}(\boldsymbol{\bullet})$. Fitting curves and the literature values for methyl acetate (1) + ethanol (2) at different pressures: (+) $101.3 \mathrm{kPa}$ (Ortega et al., 1990), and (口) $127.99 \mathrm{kPa}$ (Ortega and Susial, 1990).

The experimental data from this paper at $0.1 \mathrm{MPa}$ show that the methyl acetate + ethanol binary system has an azeotrope at $\mathrm{x}_{1 \mathrm{az} 100}=\mathrm{y}_{\mathrm{az} 100}=0.959$ and $\mathrm{T}_{\mathrm{az} 100}$ $=329.95 \mathrm{~K}$. This result does not differ significantly with the literature data (Gmehling et al., 2004) due to the fact that the small difference in temperature is within the range of error of the thermocouple used in this work. On the other hand, for 0.3 and $0.7 \mathrm{MPa}$ a modification in the azeotropic data is verified. For these isobaric conditions, the location of the singular point is $\mathrm{x}_{\text {laz300 }}=\mathrm{y}_{1 \mathrm{az} 300}=0.892, \mathrm{~T}_{\mathrm{az} 300}=364.15 \mathrm{~K}$ at $0.3 \mathrm{MPa}$; and $\mathrm{x}_{1 \mathrm{az} 700}=\mathrm{y}_{1 \mathrm{az} 700}=0.723, \mathrm{~T}_{\mathrm{az} 700}=398.25 \mathrm{~K}$ at $0.7 \mathrm{MPa}$. A representation of the experimental azeotropic data of this paper and the bibliografic data (Gmehling et al., 2004) shows a relation between both (Figure 3). Azeotropic data at 0.1 and $0.7 \mathrm{MPa}$ show a suitable position regarding the correlation equation; however, the azeotropic data at $0.3 \mathrm{MPa}$ present an error of less than $5 \%$, as shown in the inset in Figure 3.

The UNIFAC (Fredenslund et al., 1977; Fredenslund et al., 1975; Larsen et al., 1987) group contribution method and the ASOG (Kojima and

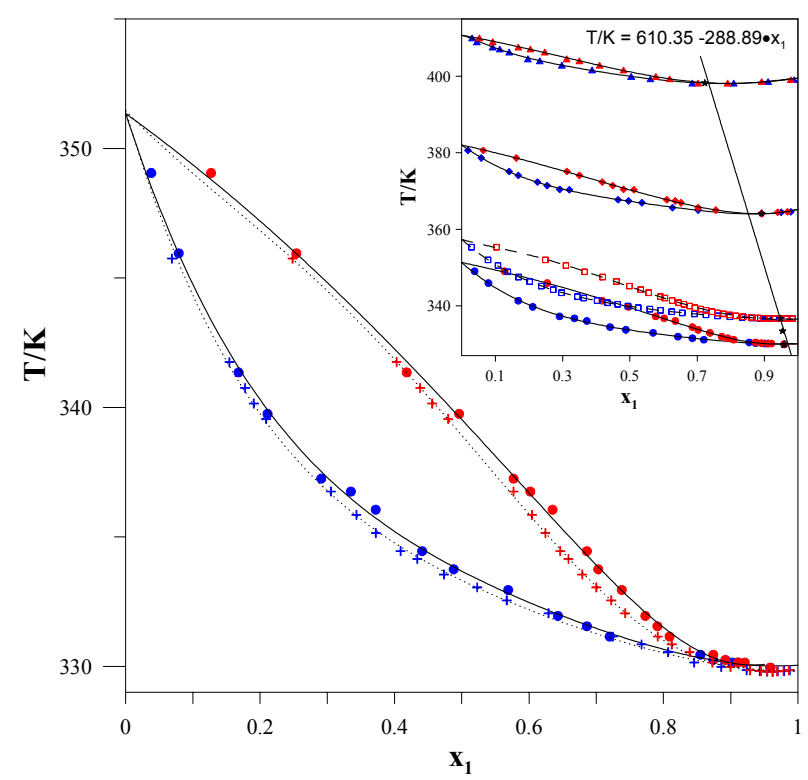

Figure 3: Experimental values and $\mathrm{FF}$ curves of $\mathrm{T}$ vs. $x_{1}$ and $T$ vs. $y_{1}$ for methyl acetate (1) + ethanol (2) at $0.1 \mathrm{MPa}(\bullet), 0.3 \mathrm{MPa}(\bullet)$ and $0.7 \mathrm{MPa}(\mathbf{\bullet})$ compared with FF curves and the literature values for methyl acetate (1) + ethanol (2) at different pressures: (+) $101.3 \mathrm{kPa}$ (Ortega et al., 1990), and (口) $127.99 \mathrm{kPa}$ (Ortega and Susial, 1990).

Tochigi, 1979) model were used in previous papers (Ortega et al., 1990; Ortega and Susial, 1990; Susial et al., 1989) for the prediction of the VLE of isobaric data at pressures near atmospheric. This paper evaluates the behaviour in the prediction of VLE data by the different versions of UNIFAC and the ASOG group contribution models with respect to the experimental data obtained in this study at moderate pressures. Results are presented in Table 5.

Table 5 show that all group contribution models return reasonably good predictions, with mean deviations near $1 \%$ in the vapor phase mole fraction. However, all models generally return a higher average error in the prediction of the activity coefficient when applied to the systems studied at 0.3 and $0.7 \mathrm{MPa}$, the average error being greater at higher pressures. A new determination of the parameters of the different models will probably be required. On the other hand, the group contribution models (Table 5) yield acceptable predictions at $0.1 \mathrm{MPa}$ for the azeotropic data. Nevertheless, at higher pressures, several models present significant discrepancy. 
Table 5: Mean deviations and average errors in the prediction of VLE data. Experimental and predicted azeotropic data

\begin{tabular}{|c|c|c|c|c|c|}
\hline & $\begin{array}{c}\text { UNIFAC } 1975 \text { (A) } \\
\text { COH/COO }\end{array}$ & $\begin{array}{c}\text { UNIFAC } 1977 \text { (B) } \\
\text { CCOH/COOC }\end{array}$ & $\begin{array}{c}\text { UNIFAC } 1987(\mathrm{C}) \\
\text { OH/COOC }\end{array}$ & $\begin{array}{c}\text { UNIFAC } 1993 \text { (D) } \\
\text { OH/COOC }\end{array}$ & $\begin{array}{c}\text { ASOG } 1979(E) \\
\text { OH/COO }\end{array}$ \\
\hline \multicolumn{6}{|c|}{ methyl acetate (1) + ethanol (2) at $0.1 \mathrm{MPa}$} \\
\hline$\delta\left(\mathrm{y}_{1}\right)$ & 0.01 & 0.01 & 0.01 & 0.01 & 0.01 \\
\hline$\overline{\mathrm{e}}\left(\gamma_{1}\right)$ & 2.71 & 3.23 & 3.55 & 2.42 & 4.39 \\
\hline$\delta(\mathrm{T}) / \mathrm{K}$ & 0.36 & 0.76 & 0.33 & 0.38 & 0.45 \\
\hline \multicolumn{6}{|c|}{ Azeotropic data } \\
\hline $\mathrm{x}_{1 \mathrm{az} \exp }=0.959$ & 0.910 & 0.907 & 0.914 & 0.908 & 0.915 \\
\hline $\mathrm{T}_{\mathrm{az} \exp }=329.95 \mathrm{~K}$ & 329.83 & 329.79 & 330.14 & 329.83 & 330.17 \\
\hline \multicolumn{6}{|c|}{ methyl acetate (1) + ethanol (2) at $0.3 \mathrm{MPa}$} \\
\hline$\delta\left(\mathrm{y}_{1}\right)$ & 0.01 & 0.01 & 0.02 & 0.01 & 0.01 \\
\hline$\overline{\mathrm{e}}\left(\gamma_{1}\right)$ & 2.79 & 4.08 & 7.47 & 3.79 & 3.59 \\
\hline$\delta(\mathrm{T}) / \mathrm{K}$ & 0.38 & 1.29 & 1.52 & 0.44 & 0.83 \\
\hline \multicolumn{6}{|c|}{ Azeotropic data } \\
\hline $\mathrm{x}_{1 \mathrm{az} \exp }=0.892$ & 0.829 & 0.780 & 0.926 & 0.844 & 0.796 \\
\hline $\mathrm{T}_{\mathrm{az} \exp }=364.15 \mathrm{~K}$ & 364.95 & 364.45 & 365.96 & 365.17 & 365.58 \\
\hline \multicolumn{6}{|c|}{ methyl acetate (1) + ethanol (2) at $0.7 \mathrm{MPa}$} \\
\hline$\delta\left(\mathrm{y}_{1}\right)$ & 0.01 & 0.02 & 0.02 & 0.01 & 0.01 \\
\hline$\overline{\mathrm{e}}\left(\gamma_{1}\right)$ & 5.75 & 11.26 & 7.27 & 3.67 & 8.25 \\
\hline$\delta(\mathrm{T}) / \mathrm{K}$ & 1.74 & 3.18 & 1.16 & 1.03 & 2.12 \\
\hline \multicolumn{6}{|c|}{ Azeotropic data } \\
\hline $\mathrm{x}_{1 \mathrm{az} \exp }=0.723$ & 0.726 & 0.678 & 0.886 & 0.748 & 0.689 \\
\hline $\mathrm{T}_{\mathrm{az} \exp }=398.25 \mathrm{~K}$ & 396.67 & 395.37 & 399.57 & 397.48 & 397.17 \\
\hline
\end{tabular}

(A) Fredenslund et al., 1975; (B) Fredenslund et al., 1977; (C) Larsen et al., 1987; (D) Gmehling et al., 1993; (E) Kojima and Tochigi, 1979

\section{CONCLUSIONS}

VLE data at moderate pressures have been obtained with a copper ebulliometer. Data for the methyl acetate + ethanol binary system at $0.1,0.3$ and $0.7 \mathrm{MPa}$ are presented. The experimental data passed positively the consistency test of Van Ness and showed satisfactory agreement with literature data. This binary system forms an azeotrope at $0.1 \mathrm{MPa}$, which has been verified via good agreement with literature data.. It has been also verified that the azeotropic data expressed as ester mole fraction decreases with a pressure increase.

The new isobaric data obtained at moderate pressures have been used to verify the predictive behavior of several group contribution models. The predictions achieved using the UNIFAC and ASOG models were verified by the determination of the mean deviations in the vapor phase mole fraction and the mean error in the estimation of the liquid phase activity coefficient. Results show that the predictions are good for the VLE at atmospheric pressure, while higher deviations were obtained with the pressure increase. When the UNIFAC and ASOG models were used for the azeotropic data prediction, the calculated values did not show a good agreement with the experimental data from this work at moderate pressures.

\section{NOMENCLATURE}

\begin{tabular}{|c|c|c|}
\hline$A_{k}$ & Parameter & \\
\hline $\mathrm{Bij}$ & $\begin{array}{l}\text { Cross second virial } \\
\text { coefficient }\end{array}$ & $\mathrm{m}^{3} \cdot \mathrm{mol}^{-1}$ \\
\hline$\overline{\mathrm{e}}$ & Average error & $\%$ \\
\hline $\mathrm{F}$ & $\begin{array}{l}\text { Property }\left(\mathrm{F}=\mathrm{y}_{1} ; \mathrm{F}=\left(\mathrm{y}_{1}-\mathrm{x}_{1}\right)\right. \\
\mathrm{F}=\gamma_{1} ; \mathrm{F}=\gamma_{2} ; \mathrm{F}=\mathrm{T} / \mathrm{K} ; \\
\left.\mathrm{F}=\mathrm{G}^{\mathrm{E}} / \mathrm{RT}\right)\end{array}$ & \\
\hline $\mathrm{G}^{\mathrm{E}}$ & Excess free energy & $\mathrm{J} \cdot \mathrm{mol}^{-1}$ \\
\hline $\mathrm{m}$ & $\begin{array}{l}\text { Number of equation } \\
\text { parameters }\end{array}$ & \\
\hline $\mathrm{n}$ & $\begin{array}{l}\text { Number of experimental } \\
\text { data }\end{array}$ & \\
\hline $\mathrm{nD}$ & Refractive index & \\
\hline $\mathrm{p}_{\mathrm{i}}^{\mathrm{o}}$ & Vapor pressure & $\mathrm{kPa}$ \\
\hline $\mathrm{p}$ & Total pressure & $\mathrm{kPa}$ \\
\hline $\mathrm{R}$ & Universal gas constant & $\mathrm{J} \cdot \mathrm{K}^{-1} \cdot \mathrm{mol}^{-1}$ \\
\hline $\mathrm{R}_{\mathrm{T}}$ & Parameter & \\
\hline $\mathrm{T}$ & Temperature & $\mathrm{K}$ \\
\hline$v_{i}^{L}$ & Liquid molar volume & $\mathrm{m}^{3} \cdot \mathrm{mol}^{-1}$ \\
\hline $\mathrm{X}$ & Liquid-phase mole fraction & \\
\hline $\mathrm{y}$ & Vapor-phase mole fraction & \\
\hline
\end{tabular}

\section{Subscripts}

$\begin{array}{ll}\text { az } & \text { Azeotrope } \\ \text { bp } & \text { Normal boiling point }\end{array}$ 
cal

$\exp$

$\mathrm{i}, \mathrm{j}$

lit

1

\section{Greek Letters}

$\delta \quad$ Mean deviation

$\phi \quad$ Fugacity coefficient

$\gamma \quad$ Activity coefficient

$\rho \quad$ Density

$\sigma \quad$ Standard deviation

\section{REFERENCES}

Barker, J. A., Determination of Activity Coefficients from Total Pressure Measurements. Australian J. Chem, 6, 207-210 (1953).

de Afonso, C., Ezama, R., Losada, P., Calama, M. A., Llanas, B., Pintado, M., Saenz de la Torre, A.F., Equilibrio Isobárico Líquido.Vapor. III Desarrollo y ensayo de un aparato de equilibrio de pequeña capacidad. Anales de Química de la RSEQ, 79, 243-253 (1983).

Fredenslund, Aa., Gmehling, J. and Rasmussen, P., Vapor-liquid Equilibria Using UNIFAC. A Group Contribution Model. Elsevier, Amsterdam (1977).

Fredenslund, Aa., Jones, R. L. and Prausnitz, J. M., Group-Contribution Estimation of Activity Coefficients in Nonideal Liquid Mixtures. AIChE J., 21, 1086-1099 (1975).

Gmehling, J., Li, J. and Schiller, M., A Modified UNIFAC Model. 2. Present Parameter Matrix and Results for Different Thermodynamic Properties. Ind. Chem. Eng. Res., 32, 178-193 (1993).

Gmehling, J., Menke, J., Krafczyk, J. and Fischer, K., Azeotropic Data. Ed. Wiley-VCH Verlag, 2 ed. Part. 1, p. 317, Weinheim (2004).

Gmehling, J. and Onken, U., Vapor-Liquid Equilibrium Data Collection. Chemistry Data Series. Vol. I, Part. 2, pp. 330-335, Dechema, Frankfurt (1986).

Hayden, J. G. and O'Connell, J. P., A generalised method for predicting second virial coefficients. Ind. Eng. Chem. Process Des. Dev., 14, 209-216 (1975).

Holmes, M. J. and Vanwinkle, M., Prediction of Ternary Vapor-Liquid. Equilibria from Binary Data. Ind. Eng. Chem., 62, 21-31 (1970).

Kojima, K. and Tochigi, K., Prediction of VaporLiquid Equilibria by the ASOG Method. Kodansha Ltd., Tokyo (1979).
Larsen, B. L., Rasmussen, P. and Fredenslund, Aa., A modified UNIFAC Group-Contribution Model for Prediction of Phase Equilibria and Heats of Mixing. Ind. Eng. Chem. Res., 26, 2274-2286 (1987)

Nagata, I., Ohta, T. and Nakagawa, S., Excess Gibss Free Energies and Heats of Mixing for Binary Alcoholic Liquid Mixtures. J. of Chem. Eng. Japan, 9, 276-281 (1976).

Nelder, J. and Mead, R., A Simplex Method for Function Minimization, Comput. J., 7, 308-313 (1967).

Ortega, J., Susial, P. and de Afonso, C., VLE Measurementes at $101.32 \mathrm{kPa}$ for Binary Mixtures of Methyl Acetate+ Ethanol or 1Propanol. J. Chem. Eng. Data, 35, 350-352 (1990).

Ortega, J. and Susial, P., VLE at 114.66 and $127.99 \mathrm{kPa}$ for the Systems Methyl Acetate + Ethanol and Methyl Acetate + Propan-1-ol. Measurements and Prediction. J. of Chem. Eng. Japan, 23, 621-626 (1990).

Ortega, J. and Susial, P., Vapor-Liquid Equilibria Behavior of Methyl Esters and Propan-2-ol at 74.66, 101.32 and $127.99 \mathrm{kPa}$. J. of Chem. Eng. Japan, 26, 259-265 (1993).

Postigo, M. A., Mariano, A. B., Jara, A. F. and Zurakoski, N., Isobaric Vapor-Liquid Equilibria for the Binary Systems Benzene + Methyl Ethanoate, Benzene + Butyl Ethanoate and Benzene + Methyl Hepthanoate at $101.31 \mathrm{kPa}$. J. Chem. Eng. Data, 54, 1575-1579 (2009).

Riddick, J. A., Bunger, W. B. and Sakano, T. K., Organic Solvents. 4th ed., Wiley-Interscience. New York (1986).

Susial, P., Ortega, J., de Afonso, C. and Alonso, C., VLE Measurements for Methyl PropanoateEthanol and Methyl Propanoate-Propan-1-ol at $101.32 \mathrm{kPa}$. J. Chem. Eng. Data, 34, 247-250 (1989).

Susial, P., Sosa-Rosario, A. and Rios-Santana, R., VLE with a New Ebulliometer: Ester+Alcohol System at $0.5 \mathrm{MPa}$. Chinese J. Chem. Eng., 18, 1000-1007 (2010).

Van Ness, H. C., Byer, S. M. and Gibbs, R. E., Vapor-Liquid Equilibrium: I. An Appraisal of Data Reduction Methods. AIChE J., 19, 238-244 (1973).

Yaws, C. L., Yaw's Handbook of Thermodynamic and Physical Properties of Chemical Compounds. Ed Knovel, Norwich-New York (2003).

Yen, L. C. and Woods, S. S., A generalized equation for computer calculation of liquid densities. AIChE J., 12, 95-99 (1966). 